\title{
Experiments on pattern formation in reacting systems with chaotic advection
}

\author{
T. H. Solomon ${ }^{*}$, M. S. Paoletti ${ }^{\dagger}$ and C. R. Nugent \\ Department of Physics and Astronomy, Bucknell University, Lewisburg, PA 17837 USA
}

\begin{abstract}
We present the results of experiments on advection-reaction-diffusion processes. Two flows are studied: a blinking vortex flow and a chain of alternating vortices. Mixing in both of these flows has been shown to be chaotic in general. The fluid is composed of the chemicals for the Belousov-Zhabotinsky (BZ) chemical reaction. We investigate the effects of chaotic mixing on the patterns that form in this system. Three experiments are described: (a) pattern formation in the oscillatory BZ reaction; (b) front propagation and mode-locking for the excitable BZ reaction in an oscillating vortex chain; and (c) synchronization of a network of fluid oscillators by superdiffusive transport and Lévy flights. The experiments are complemented by numerical simulations that illustrate the chaotic transport of these flows.
\end{abstract}

\section{Introduction}

For several decades, there has been a significant amount of interest in patterns that form in reaction-diffusion systems [1],[2], e.g., chemically-reacting or biological systems without any fluid flows. But most fluid systems are not stagnant; rather, there are typically flows, and these flows dramatically affect the mixing properties of the system. This, in turn, has a significant effect on the pattern formation process since reaction of different species in the flow is limited by mixing. The problem is particularly interesting in light of recent studies that have shown that mixing can be chaotic, even for simple, laminar fluid flows. The general advection-reaction-diffusion problem has recently begun to receive theoretical and numerical attention, particularly in the regime where mixing is chaotic [3],[4],[5],[6],[7],[8], [9]. However, there have been very few experimental studies of advection-reaction-diffusion systems. In this article, we review some experiments that we have conducted on pattern-

\footnotetext{
* Corresponding author. Tel: +1-570-577-1348; fax +1-570-577-3153

E-mail address: tsolomon@bucknell.edu.

${ }^{\dagger}$ Current address: Department of Physics, University of Maryland, College Park, MD USA
} 
formation processes in advection-reaction-diffusion systems with chaotic advection. Three sets of experiments are described: (1) Pattern formation of an oscillatory chemical reaction in a flow with chaotic mixing [10]; (2) front propagation and mode-locking for an advancing chemical reaction in an oscillating vortex chain [11],[12]; and (3) synchronization of chemical oscillators in a fluid flow via superdiffusive transport [13].

\section{Pattern formation in a blinking vortex flow}

In 1984, Aref demonstrated [14] that mixing is typically chaotic in a simple, laminar fluid flow composed of two point vortices that blink on and off periodically (Figure 1a). We have built an experiment that generates this flow in a simple, table-top apparatus. The flow is generated via a magnetohydrodynamic technique [10],[15] (Figure 1b). An electrical current passes radially through a thin layer of an electrolytic solution (either dilute sulfuric acid or the chemicals for the Belousov-Zhabotinsky reaction). This current -- which converges at one of two center electrodes -- interacts with a strong magnetic field produced by a Nd-Fe-Bo magnet below the fluid layer. The result is a flow that circles around that particular electrode. Blinking of the vortices is achieved by alternating periodically between the two center electrodes. The result is a flow that alternates between circling around one vortex and circling around the other.

(a)

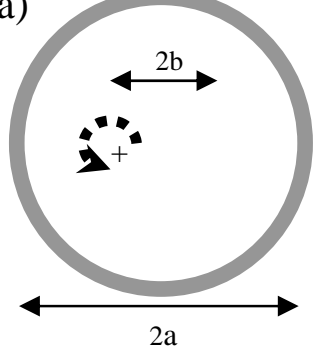

(b)

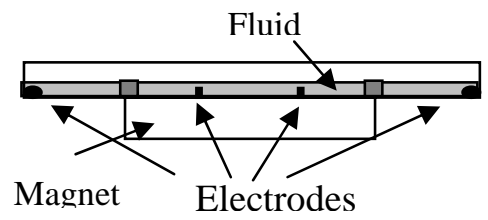

Figure 1. (a) Blinking vortex flow. The fluid alternates between circling around the left vortex and around the right vortex. (b) Apparatus used to generate the flow. An electrical current passes radially through a thin layer of an electrolytic solution, converging on one of two electrodes in the middle. The current interacts with a magnetic field produced by a permanent magnet, resulting in a flow that circles around the electrode.

The fluid is composed of the chemicals typically used for the Belousov-Zhabotinsky (BZ) reaction [16],[17], a chemical reaction that is well-known for its oscillatory (and sometimes chaotic) time dependence when well-mixed. (See Reference 10 for details about the chemicals used in this reaction.) In the absence of any fluid flow, the reaction forms a pattern of spirals and/or bulls-eye patterns, typical of reaction-diffusion systems (Figure 2a).

With the flow turned on, the patterns change dramatically (Figures 2b, c and d). For these images, the blinking period $T$ and the flow amplitude $A$ vary; however, the dimensionless blinking period $\mu$ - which is the product of $A$ and $T$, divided by the square of the radius of the system - is held fixed. The dimensionless blinking period determines the mixing patterns that form, but does not determine how long it takes for those patterns to form. With decreasing $T$, the flow mixes faster and faster. In Reference 10, we define a mixing time 
determined by the interplay between advective mixing and molecular diffusion; conceptually, this is the time that it takes for chaotic mixing to stretch elements in the flow into tendrils that are thin enough for molecular diffusion to finish the mixing.

Figures $2 \mathrm{e}, \mathrm{f}, \mathrm{g}$ and h show mixing fields determined numerically for the same conditions as for Figures $2 \mathrm{a}-\mathrm{d}$. These fields are obtained by simulating the motion of triplets of tracers, initially very close to each other. At each time step, the mixing field at a point is defined as the ratio of the largest separation between the tracers in the triplet divided by the initial separation. It is apparent when comparing Figures $2 \mathrm{~b}$-d with Figures $2 \mathrm{f}$-h that for the advection-reaction-diffusion case, the mixing fields do a good job of capturing the dominant pattern formation behavior for the oscillatory reaction. (The only exception is for the reaction-diffusion limit for which the mixing fields cannot capture the patterns.)

The implication of these studies is that pattern formation in a reacting system with chaotic mixing can be understood quite well by considering almost purely the mixing behavior of the system without much regard to the details of the reaction itself. We expect, therefore, that mixing fields should be able to capture the dominant pattern formation process for a wide range of advection-reaction-diffusion systems.

More details about these experiments can be found in Reference 10.

(a)

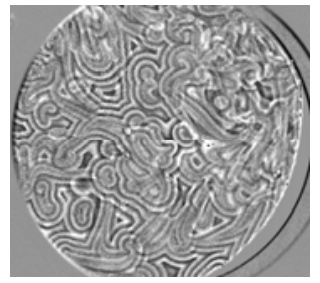

(e)

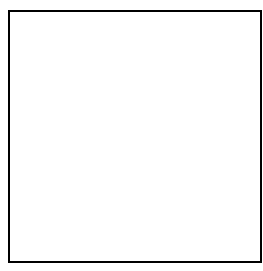

(b)

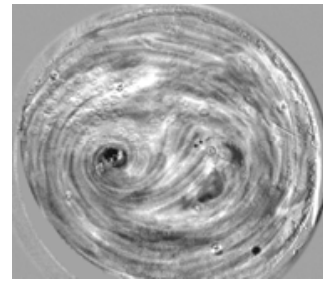

(f)

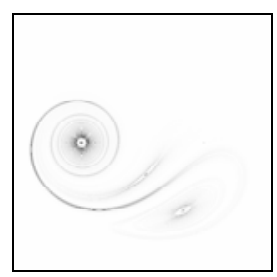

(c)

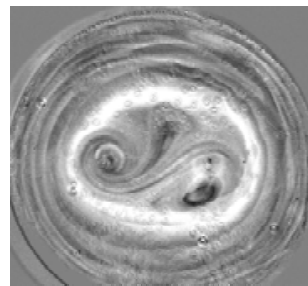

(g)

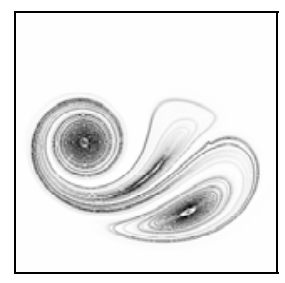

(d)

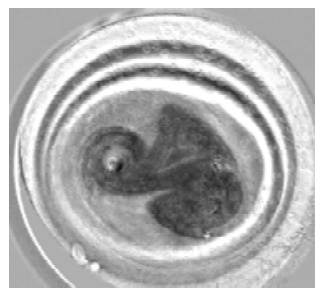

(h)

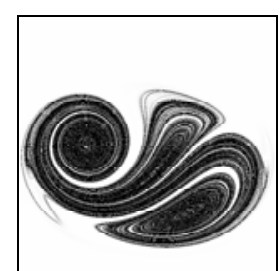

Figure 2. (a)-(d) Images of Belousov-Zhabotinsky patterns in blinking vortex flow. For all cases, the non-dimensional blinking period $\mu=0.52$ (see Reference 10 for definition). (e)-(h) Mixing fields for the same flows. (a) and (e): no flow. The blinking frequency is $0.010 \mathrm{~Hz}$ for (b) and (f), $0.030 \mathrm{~Hz}$ for (c) and (g), and $0.050 \mathrm{~Hz}$ for (d) and (h). 


\section{Front propagation and mode-locking}

There are numerous processes that are governed by the growth of one species in the system at the expense of another species. The interface between the two regions is often referred to as a front, and there has been a significant amount of interest in the manner in which this front moves in reaction-diffusion systems. In fact, there is a well-known theory by Fisher and Kolmogorov [18],[19] that predicts a definitive value for the front propagation speed, given information about the reaction kinetics and the molecular diffusion coefficient. The question arises as to how front propagation is affected by the presence of fluid flows in the system, particularly in situations where the mixing is chaotic. Chaotic mixing processes can often be quantified as enhanced diffusion, so it is natural to predict that the FisherKolmogorov (FK) theory will still work as long as the molecular diffusion coefficient in the theory is replaced with the enhanced diffusion coefficient. Recent theoretical/numerical studies [5], however, have indicated that a simple extension of the FK theory does not necessarily work for cellular flows with periodic time dependence. Specifically, these studies showed that mode-locking is possible for front propagation in an oscillating chain of vortices (Figure 3), a flow which had been shown previously [15],[20],[21] to display chaotic mixing.
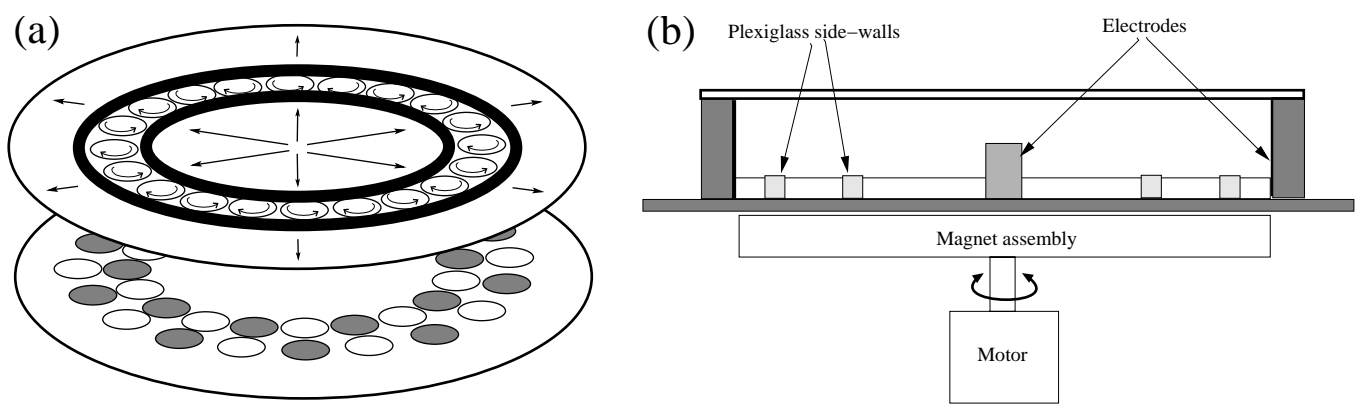

Figure 3. (a) Exploded view of magnetohydrodynamic forcing and resulting flow, composed of an annular chain of 20 counter-rotating vortices. (b) Side view of experimental apparatus.

An electrical current passes radially through a thin layer of an electrolytic solution. This current interacts with an alternating magnetic field produced by two rings of magnets mounted in an assembly below the fluid layer. The magnet assembly is mounted coaxially on a motor whose motion can be controlled to oscillate, drift with a constant angular velocity, or move with a combination of both drift and oscillations.

We have reproduced some of these numerical simulations to illustrate locking phenomena [12]. Mode-locking is defined for a propagating front as follows: when modelocked, the front advances an integer number $N$ of wavelengths in an integer number $M$ of drive periods. Figure 4(a) shows a numerical sequence showing a $(N, M)=(1,1)$ mode such that the front advances two vortex widths ( 1 wavelength of the flow) in 1 drive period. Figure 4(b) shows a numerical sequence for a $(1,2)$ mode-locked state. 
(a)

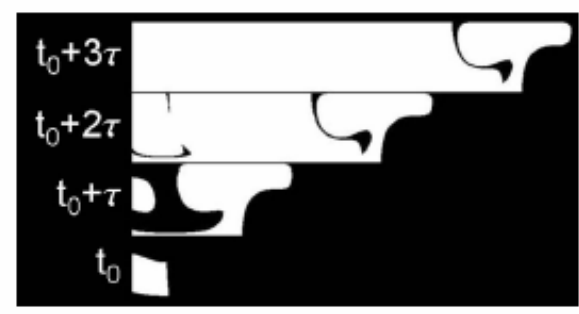

(b)

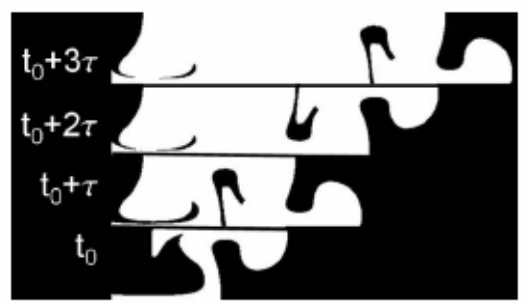

Figure 4. Simulations showing mode-locking with $(N, M)=(1,1)$ for $(a)$ and $(1,2)$ for (b).

We have tested these predictions with experiments on front propagation in an oscillating vortex chain [11],[12]. The flow and apparatus are show in in Figure 3. There are 20 vortices in the vortex chain, which is oriented in an annular configuration. As with the blinking vortex flow described in Section II, the vortex chain is forced magnetohydrodynamically. Two rings of $20 \mathrm{Nd}-\mathrm{Fe}-\mathrm{Bo}$ magnets are set in a magnet assembly, above which rests a thin layer of an electrolytic solution. The magnets alternate in polarity, as shown in Figure 3. An electrical current passes radially through the fluid layer and interacts with the alternating magnetic field. The result is a chain of 20 alternating vortices in the fluid layer. The magnet assembly is mounted co-axially on a motor which can be programmed to rotate in an oscillatory pattern, with a constant drift velocity, or with a combination of drift and oscillatory terms; the vortex chain itself moves with the magnet assembly. For the experiments discussed in this section, the magnet assembly is programmed to oscillate sinusoidally, although the frequency and amplitude of the oscillations are varied.

The chemical reaction used is an excitable version of the Belousov-Zhabotinsky reaction. (See References 11 and 12 for details of the chemistry.) The chemicals are initially orange in color, but a green front can be triggered by inserting a silver wire into the flow momentarily. Once initiated, the front propagates across the system. The reaction is actually a "pulse" rather than a front - the green reacting zone is followed by a return of the system to the orange state behind the leading edge. In the absence of any flow at all, the result is a green

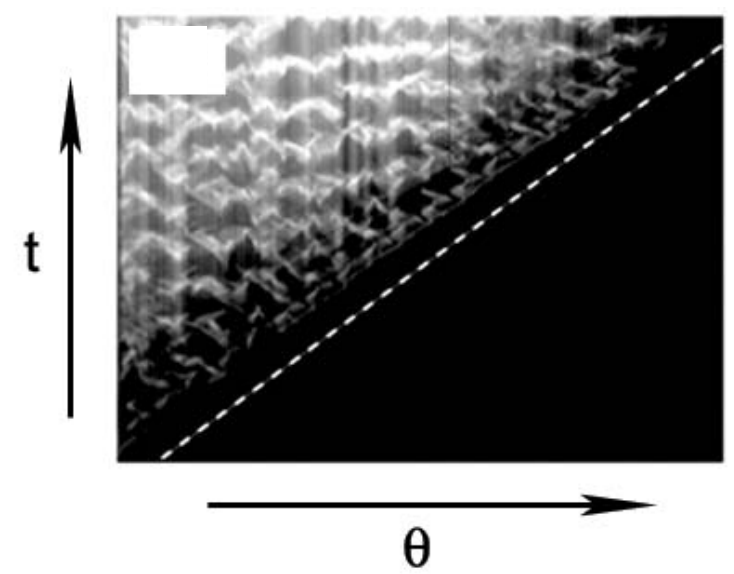

Figure 5. Space-time plot for an experimental run that is mode-locked with $(\mathrm{N}, \mathrm{M})=(1,2)$. 
ring that propagates outward with the rest of the system remaining orange. The orange section can be re-triggered multiple times, either if the green front returns due to flows in the system or with the re-insertion of a silver wire. The reaction is also photo-sensitive and can be inhibited by strong illumination with blue and green light. In the experiments, a region covering two vortex widths is illuminated, and the reaction is triggered next to this region. The reaction can then propagate only in one direction around the annulus. The duration of the experiment can be extended by following the front with the blinding region, erasing the region behind the advancing front and enabling the pulse to travel multiple times around the annulus.

In the absence of any periodic time dependence, the front advances through the system due to several factors: (a) advection carries the front around a vortex; (b) the front "burns" its way inward toward the vortex centers; and (c) when the front reaches a corner of a vortex, it "burns" across the separatrix between adjacent vortices and triggers the reaction in the next vortex.
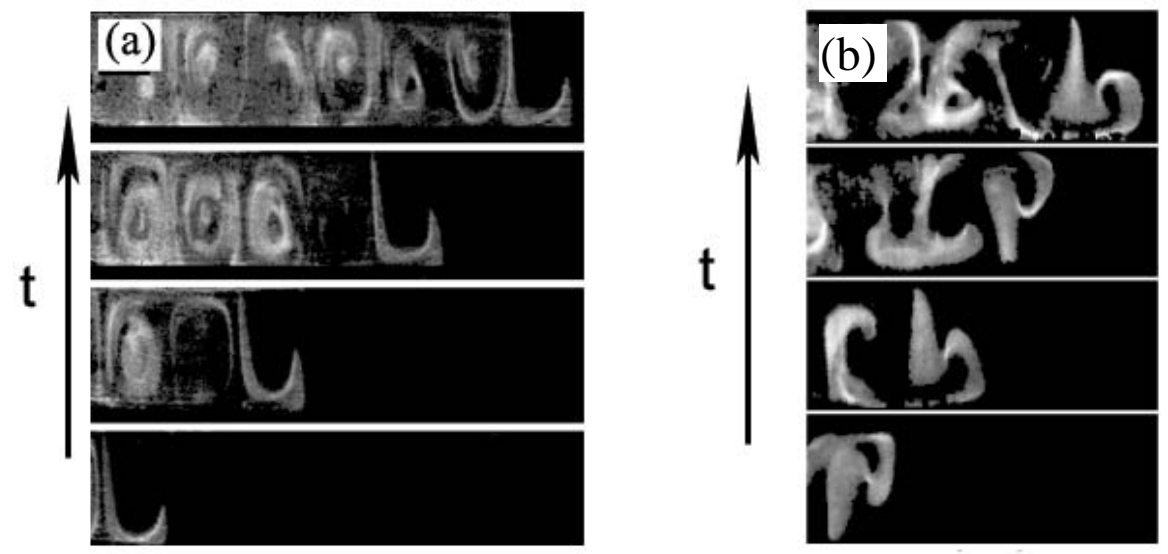

Figure 6. Experimental sequences showing mode-locking with $(\mathrm{N}, \mathrm{M})=(1,1)$ for $(\mathrm{a})$ and $(1,2)$ for (b).

When the system is forced with oscillatory time dependence, the front propagation shows many of the characteristics of chaotic mixing in the oscillating vortex chain. Despite the chaotic behavior, though, the front typically (for large enough amplitude) propagates with a constant velocity, as shown in the spacetime plot of Figure 5. Sequences of images (Figure 6 ) show that the system is showing mode-locking; compare Figure 6a with Figure 4a and Figure $6 \mathrm{~b}$ with Figure $4 \mathrm{~b}$. The propagation speed can be determined from the slope of the spacetime plot (Figure 5). If plotted as a function of oscillation frequency (Figure 7), two dominant modes are apparent $-\mathrm{a}(1,1)$ and $(1,2)$ mode. When locked, the propagation speed grows linearly with frequency.

A parameter space diagram (Figure 8) reveals the conditions for which the fronts are mode-locked. For small amplitudes of oscillation, the system does not mode-lock - the front speed fluctuates in time and there is no repeating pattern. For larger amplitudes, locking has been found for both the $(1,1)$ and $(1,2)$ locking tongues, and there is a region of overlap where the front switches alternately between the $(1,1)$ and $(1,2)$ modes during a single run.

These results agree with the predictions of Reference 5 . The fact that the system modelocks indicates clearly that a simple extension of the FK theory is not valid, and that a new theoretical treatment is needed to predict front propagation in an advection-reaction-diffusion 
system. In particular, the role of coherent structures (vortices, in this case) needs to be assessed. Our expectation is that deviations from FK predictions will be common, potentially even for turbulent flows, since coherent flow structures are quite common in many natural fluid flows.

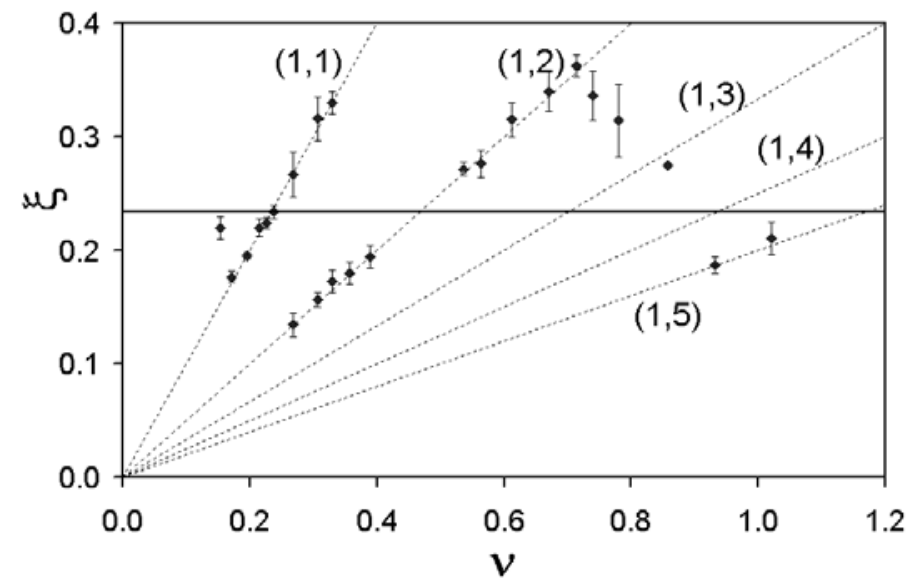

Figure 7. Experimental results showing non-dimensional front speed $\xi$ as a function of non-dimensional frequency $v$. The dotted lines show the theoretical predictions (with no fitted parameters) for mode-locked speeds.

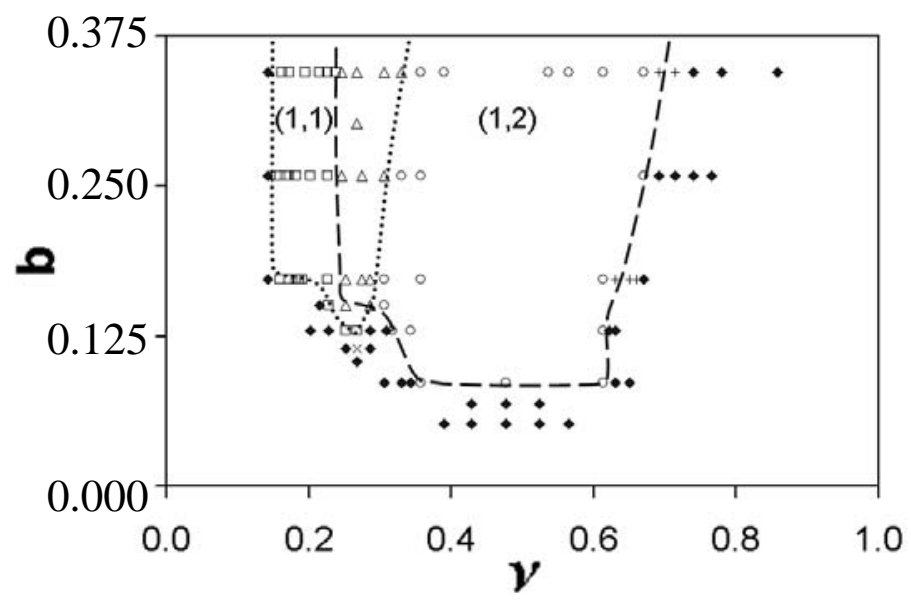

Figure 8. Parameter-space plot showing Arnol'd tongues for $(1,1)$ and $(1,2)$ mode-locked states. Filled diamonds denote unlocked states, whereas open squares, open circles and open triangles denote states with $(1,1),(1,2)$ and combination $(1,1) /(1,2)$ mode-locking, respectively.

More details about these experiments can be found in References 11 and 12 . 


\section{Synchronization of a continuous network of oscillators}

The third set of experiments [13] deals with synchronization of chemical oscillators. There has been a tremendous amount of research recently into networks of oscillators and how they synchronize. Those studies were energized in the past few years by a theoretical study [22] that showed that network connectivity could be enhanced significantly in a SmallWorld Network with random short-cuts that connect distant parts of the network, in addition to regular, nearest-neighbor connections.

Our experiments consider synchronization in a continuous (rather than discrete) fluid network in which fluid mixing is the dominant mechanism of communication in the network. The flow is the same alternating vortex chain discussed in Section III, except that in addition to periodic oscillations, the vortex chain can also drift. If there are no oscillations but there is a drift, a snake-like region forms that winds around and between the vortices (Figure 9a). A tracer in this snake region rapidly moves between vortices and can traverse a long distance in a very short period of time. If the vortex chain has both oscillatory and drifting motion, then there can be a combination of both ordered and chaotic trajectories. If the drift velocity $v_{d}$ is greater than the maximum oscillation velocity $v_{o}$, then the snake region (or portions of it) is maintained (Figure 9b). In this regime, tracers in a chaotic region alternately rotate within a vortex, move between one vortex and the next, or stick to the snake region and undergo rapid motion to a distant vortex.

(a)

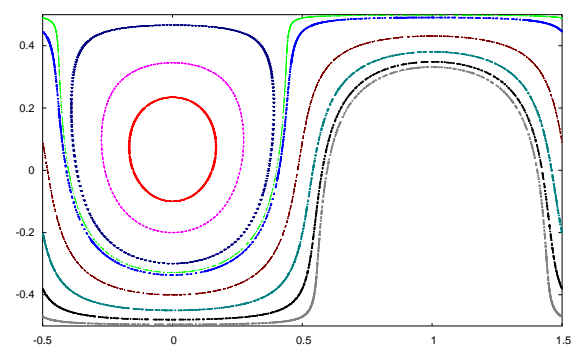

(b)

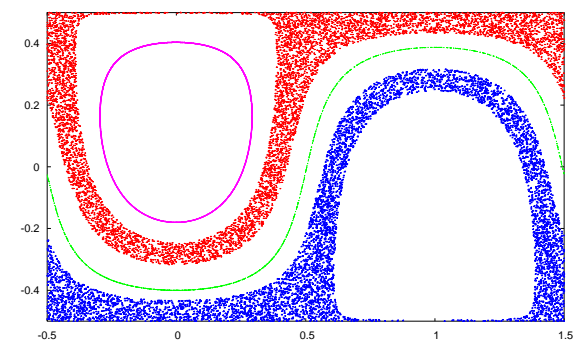

Figure 9. Transport in the drifting vortex chain (a) without oscillations and (b) with oscillations. In the absence of any oscillations, all trajectories are ordered and the flow is divided into two types of regions, one in which tracers rotate within a vortex core, and the other in which tracers move rapidly around and between vortices in a snake-like jet region. If there is oscillatory time dependence as well, chaotic regions forms, denoted by erratic pattern of dots in (b).

Trajectories such as this that alternately stay within a localized region and undergo rapid long-range jumps are referred to as Lévy flights [23],[24] (depending on the statistics of the jumps), and transport in this case is typically superdiffusive with a variance that grows faster than linearly in time. This is in contrast to normal, enhanced diffusion with a linear growth in the variance. The result is a system that typically shows normal diffusion for $v_{d}<v_{o}$ and superdiffusion for $v_{d}>v_{o}$ (Figure 10).

We contend that Lévy flights and superdiffusion play a role in a fluid system similar to the role of short-cuts in a Small World network. We have investigated this experimentally by studying synchronization of the oscillatory BZ reaction (with the same chemistry as in Section I) in the oscillating/drifting vortex chain. In the absence of any time-dependence (i.e., a stationary vortex chain), each vortex acts like an isolated BZ reactor with only 
minimal communication, since transport between vortices is purely via molecular diffusion. If the flow is time dependent with $v_{d}<v_{o}$, there is chaotic mixing between adjacent vortices, and the system spontaneously forms traveling waves (Figure 11a), although the traveling waves evolve in a very complicated manner as a function of time. For time-dependent forcing with $v_{d}>v_{o}$, the system typically synchronizes in one of two different ways. In most cases (Figure 11b), co-rotating synchronization is observed where the odd vortices synchronize with each other and the even vortices synchronize with each other, but there is an arbitrary phase different between these two sets of vortices. In some cases, however, the system synchronizes globally with all the vortices blinking red/blue in unison (Figure 11c).

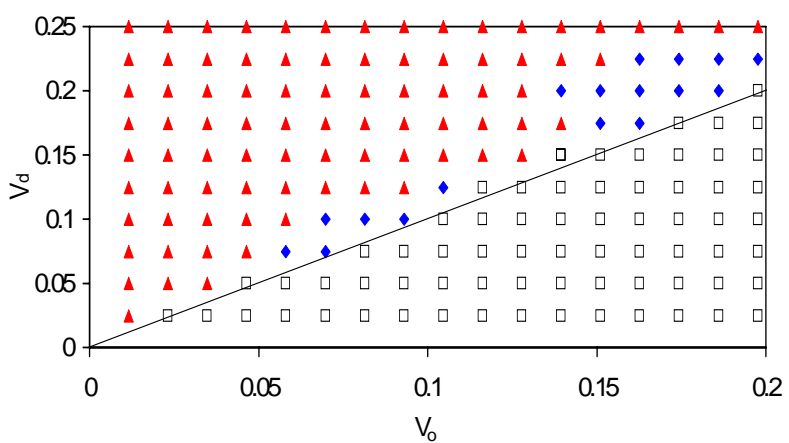

Figure 10. Parameter space plot showing regions of normal (open squares) and superdiffusive transport (filled triangles and diamonds) for the oscillating and drifting vortex chain. Two types of superdiffusive regimes are denoted, one characterized by two chaotic regions separated by an ordered jet region (triangles) and the other denoted by one chaotic region with flight-producing islands (diamonds). The solid line corresponds to $v_{d}=v_{o}$.

The synchronization behavior is summarized with a parameter space plot in Figure 12. Comparing Figure 12 with Figure 10, it can be seen that the system typically synchronizes if the transport is superdiffusive with Lévy flight trajectories, similar to the small-world networks for discrete networks.

The implication of these results is that superdiffusive transport may be a necessary (although probably not sufficient) condition for synchronization in an extended fluid system, i.e., one in which the total system size is appreciably larger than characteristic length scales of the fluid flow. It is also intriguing to consider Lévy flights in these studies as playing a role similar to the "short-cuts" in the Small World networks. But there are differences as well. First, Lévy flights in the vortex chain are not random; rather, they connect every vortex to every other one with a magnitude that decays algebraically (instead of exponentially) with separation. Second, the amount of fluid participating in the flights depends on the sizes of ordered regions in the flow, something that has not been analyzed in detail yet.

We also speculate that techniques used to predict synchronization in a continuous fluid flow could be applied to discrete systems with large numbers of nodes, especially if the nodes are mobile. For a moving population of people, for example, it might be possible to treat the population as a "fluid" and measure its transport properties. Synchronization of various processes in this population (e.g., disease outbreak) might then be predicted by determining whether mixing in the population is diffusive or superdiffusive. 
(a)

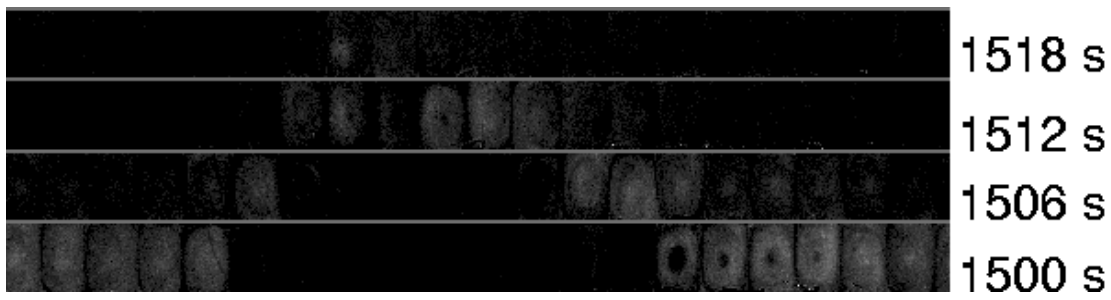

(b)

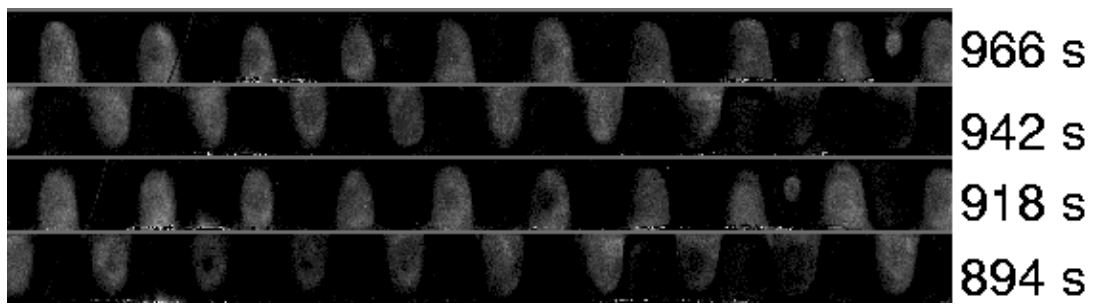

(c)

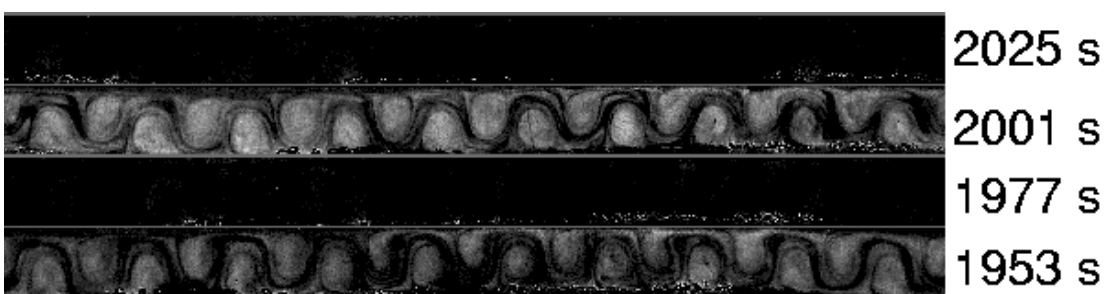

Figure 11. Sequences of images of BZ reaction in oscillating/drifting vortex chain. (a) Wave behavior seen when $v_{d}<v_{o}$. (b) Co-rotating and (c) global synchronization for $v_{d}>v_{o}$.

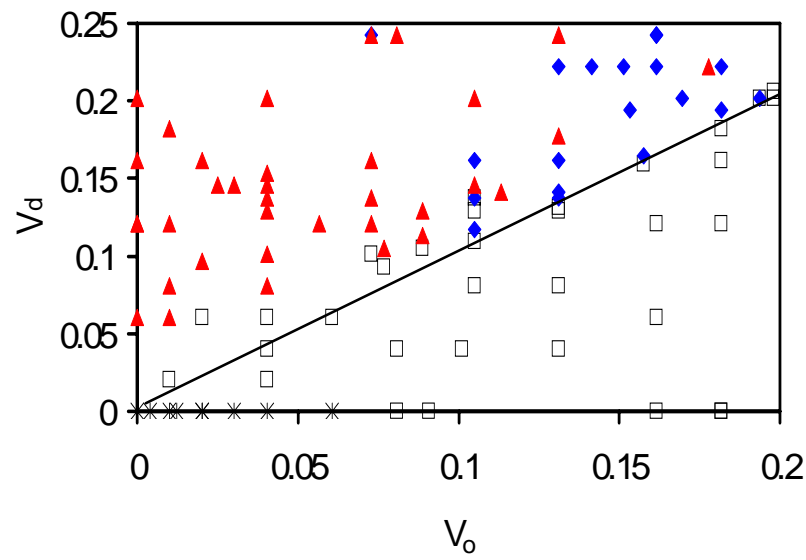

Figure 12. Parameter space diagram showing the different types of behavior for the BZ reaction in the oscillating/drifting vortex chain. The asterisks correspond to completely desynchronized states. The open squares denote traveling wave states. The filled triangles and diamonds correspond to co-rotating and globally synchronized states, respectively. The solid line corresponds to $v_{d}=v_{o}$. 


\section{Continuing work}

Numerous issues remain unanswered in advection-reaction-diffusion processes. We are currently conducting several additional experimental investigations on subject: (a) the effects of superdiffusive transport on front propagation processes; (b) the growth of a chemical region in a flow with chaotic mixing; and (c) chaotic traveling waves in an advectionreaction-diffusion system.

\section{Acknowledgements}

This work was supported by the US National Science Foundation Grants DMR-0404961 and REU-0097424.

\section{References}

[1] Grindrod, P. The Theory and Applications of Reaction-Diffusion Equations: Patterns and Waves (Clarendon Press, Oxford, 1996).

[2] Ben-Avraham, D \& Havlin, S. Diffusion and Reactions in Fractals and Disordered Systems (Cambridge University Press, Cambridge, 2000).

[3] Tel, T., de Moura, A., Grebogi, C. \& Karolyi, G. Chemical and biological activity in open flows: A dynamical system approach. Phys. Rep. 413, 91-196 (2005).

[4] Abel, M., Celani, A., Vergni, D. \& Vulpiani, A. Front propagation in laminar flows. Phys. Rev. E 64, 046307 (2001).

[5] Cencini, M., Torcini, A., Vergni, D. \& Vulpiani, A. Thin front propagation in steady and unsteady cellular flows. Phys. Fluids 15, 679-688 (2003).

[6] Karolyi, G., Pentek, A., Toroczkai, Z., Tel, T. \& Grebogi, C. Chemical or biological activity in open chaotic flows. Phys. Rev. E 59, 5468-5481 (1999).

[7] Karolyi, G., Pentek, A., Scheuring, I., Tel, T. \& Toroczkai, Z. Chaotic flow: The physics of species coexistence. Proc. Nat. Acad. Sci. U.S.A. 97, 13661-13665 (2000).

[8] Neufeld, Z. Excitable media in a chaotic flow. Phys. Rev. Lett. 87, 108301 (2001).

[9] Neufeld, Z., Kiss, I. Z., Zhou, C. \& Kurths, J. Synchronization and oscillator death in oscillatory media with stirring. Phys. Rev. Lett. 91, 084101 (2003).

[10] Nugent, C. R., Quarles, W. M. \& Solomon, T. H. Experimental studies of pattern formation in a reactionadvection-diffusion system. Phys. Rev. Lett. 93, 218301 (2004).

[11] Paoletti, M. S. \& Solomon, T. H. Experimental studies of front propagation and mode-locking in an advection-reaction-diffusion system. Europhys. Lett. 69, 819-825 (2005).

[12] Paoletti, M. S. \& Solomon, T. H. Front propagation and mode-locking in an advection-reaction-diffusion system. Phys. Rev. E 72, 046204 (2005).

[13] Paoletti, M. S., Nugent, C. R. \& Solomon, T. H. Synchronization of Oscillating Reactions in an Extended Fluid

System. Phys. Rev. Lett., in press (2006).

[14] Aref, H. Stirring by chaotic advection. J. Fluid Mech. 143, 1-21 (1984).

[15] Solomon, T. H., Tomas, S. \& Warner, J. L. The Role of Lobes in Chaotic Mixing of Miscible and Immiscible Impurities. Phys. Rev. Lett. 77, 2682-2685 (1996).

[16] Winfree, A.T. Spiral waves of chemical activity. Science 175, 634-636 (1972).

[17] Showalter, K. Pattern-formation in a ferroin-bromate system. J. Chem. Phys. 73, 3735-3742 (1980).

[18] Kolmogorov, A. N., Petrovskii, I. G. \& Piskunov, N. S. Moscow Univ. Math. Bull. (Engl. Transl.) 1, 1 (1937).

[19] Fisher, R. A. Proc. Annu. Symp. Eugen. Soc. 7, 355 (1937).

[20] Solomon, T. H. \& Gollub, J. P. Chaotic particle transport in time-dependent Rayleigh-Bénard convection. Phys. Rev. A 38, 6280-6286 (1998).

[21] Camassa, R. \& Wiggins, S. Chaotic advection in a Rayleigh-Bénard flow. Phys. Rev. A 43, 774-797 (1991). 
[22] Watts, D. J. \& Strogatz, S. H. Collective dynamics of 'small-world' networks. Nature. 393, 440-442 (1998).

[23] Shlesinger, M.F., Zaslavsky, G. M. \& Klafter, J. Strange kinetics. Nature 363, 31-37 (1993).

[24] Solomon, T. H., Weeks, E. R. \& Swinney, H. L. Observation of anomalous diffusion and Lévy flights in a two-dimensional rotating flow. Phys. Rev. Lett. 71, 3975-3978 (1993). 